\title{
Depression and Mentalizing: A Psychodynamic Therapy Process Study
}

\author{
Kari Halstensen ${ }^{1,2}$, Rolf Gjestad ${ }^{3}$, Patrick Luyten ${ }^{4,5}$, Bruce Wampold ${ }^{1,6}$, Pehr Granqvist ${ }^{7}$, \\ Gry Stålsett ${ }^{1,2}$, and Sverre U. Johnson ${ }^{1,8}$ \\ ${ }^{1}$ Modum Bad Research Institute \\ ${ }^{2}$ MF Norwegian School of Theology, Religion and Society, Oslo, Norway \\ ${ }^{3}$ Division of Psychiatry, Haukeland University Hospital, Bergen, Norway \\ ${ }^{4}$ Faculty of Psychology and Educational Sciences, KU Leuven (University of Leuven) \\ ${ }^{5}$ Research Department of Clinical, Educational and Health Psychology, UCL (University College London) \\ ${ }^{6}$ Department of Counseling Psychology, University of Wisconsin-Madison \\ ${ }^{7}$ Department of Psychology, Stockholm University \\ ${ }^{8}$ Department of Psychology, University of Oslo
}

\begin{abstract}
The present study aimed to explore the relationship between changes in depressive symptoms and the capacity to mentalize over the course of a 3-month inpatient psychodynamic therapy in a sample of 56 patients with depression. Depressive symptoms and mentalizing were assessed weekly during treatment and at 1-year follow-up with the Beck Depression Inventory and the Reflective Functioning Questionnaire (RFQ). Data were analyzed using Latent Growth Curve (LGC) modeling with structured residuals. In the total sample, depressive symptoms improved on average from baseline to the end of treatment, while mentalizing skills did not. However, individual variations were observed in mentalizing skills, with some patients improving while others did not. Within-patient residual changes in mentalizing skills did not predict residual changes in depressive symptoms. Accordingly, the results did not support mentalizing as a mechanism of change at this level. Nonetheless, between-patient effects were found, showing that patients with higher levels of mentalizing at baseline and patients whose mentalizing skills improved over the course of therapy also had greater reductions in depressive symptoms. We suggest that the presence of relatively higher mentalizing skills might be a factor contributing to moderately depressed individuals' ability to benefit from treatment, while relatively poor or absent mentalizing capacity might be part of the dynamics underlying treatment resistance in individuals with severe depression.
\end{abstract}

\section{Public Significance Statement}

Emotional and relational changes related to psychodynamic therapy are likely to be followed by reductions in depressive symptoms over the course of therapy. Between-person improvements in mentalizing were related to reductions in depressive symptoms, but within-person change in mentalizing was not found to be a mechanism of change in depression. Further research is needed to examine the role of mentalizing skills as a factor contributing to the depressed individual's ability to profit from treatment.

Keywords: depression, psychodynamic therapy, mentalizing, reflective functioning, process-outcome

This article was published Online First August 5, 2021.

Kari Halstensen (D) https://orcid.org/0000-0001-5395-5730

Sverre U. Johnson (DD https://orcid.org/0000-0001-7190-4187

The authors pay their gratitude to Hege Angeltveit and Harald Nydal for their work with the adherence test and to Patrick Curran for helpful correspondence concerning the statistical analyses. The authors also want to express their appreciation to administrative consultant Amina Kaggestad for controlling the data set before analysis, and graphical designer Grete E. Westerlund for improvements of the layout. Professor and coauthor Patrick Luyten is involved in the development, training, and dissemination of Mentalization-Based Treatment (MBT). There has been no prior dissemination of the content of this article.

Correspondence concerning this article should be addressed to Kari Halstensen, Modum Bad Research Institute, Badeveien 287, Vikersund 3370, Norway. Email: kari.halstensen@modum-bad.no
Since their inception, both cognitive-behavioral and psychodynamic formulations have centrally emphasized the role of mental representations or cognitive-affective schemas in explaining vulnerability to psychopathology in general and depression in particular (Beck et al., 1979; Blatt, 1974, 2004). Yet, more recent psychodynamic and cognitive-behavioral approaches have shifted their focus to the role of impairments in metacognition or mentalizing (also referred to as Reflective Functioning; RF) in depression and other disorders (Luyten, Fonagy, et al., 2019; Segal \& Teasdale, 2018; Watkins \& Teasdale, 2004). Rather than focusing on the content of cognitiveaffective schemas in depression, these approaches focus on the metacognitive processes involved in reflecting on self and others.

From a psychodynamic perspective, impairments in RF or mentalizing - that is, the capacity to envision the self and others 
in terms of mental states such as feelings, wishes, desires, values, and goals - are thought to play a central role in depression and suicide (Luyten et al., 2013; Luyten, Fonagy, et al., 2019). This is consistent with the so-called third-wave cognitive approaches focusing on metacognitive awareness and mindfulness in depression and its treatment. These approaches share similar emphases in focusing on distorted cognitive-affective schemas in depression and suicide and may provide a more comprehensive account of the subjective experiences of patients with depression and their problems with overcoming depressive feelings and thoughts in particular.

As conceptualized in the mentalizing perspective, depressive feelings and thoughts are rooted in reactions to threats against the individual's attachment relations. Threatened attachment relations can result in separation, rejection, and loss as well as failure experiences (Luyten, Fonagy, et al., 2019. These threats produce depressive mood, which in turn generates a loss of mentalizing capacity as perceptions are narrowed and there might be an increase in arousal due to the threats of separation and/or failure. Limited range of perception and increased arousal both reduce and obstruct the reflective processes. Hence, the loss of mentalization aggravates the depressive mood, and the result is a vicious circle that might produce a depressive condition (Luyten, Fonagy, et al., 2019).

In this circle of accumulating depressive symptoms, distortions in the mentalizing processes also play a significant role as negative biases in the individual's perceptions of self, others, and relationships might prevent the reflection needed to regulate emotions and relations (Holmes, 2009; Jurist, 2018). Negative biases in perception caused by the adult individual's failure to mentalize are related to lack of appreciation of the opaqueness of their own or someone else's mental state (Luyten, Fonagy, et al., 2019). These mentalizing failures can be considered either as hypermentalizing or as hypomentalizing (Fonagy et al., 2016; Jurist, 2018). When hypomentalizing the individual underrates the possibility to understand his or her mental processes and thereby produces an exaggerated uncertainty about the content of thoughts, feelings, and attitudes behind behavior (Bateman \& Fonagy, 2015). In the opposite perspective, hypermentalizing implies an overestimate of one's capacity for interpretations of mental states, resulting in an overstated certainty about the matter of human motivation (Fonagy et al., 2016; Jurist, 2018). Hypomentalizing might generate a sense of apathy as the individual's anticipated possibility of influence is diminished. Hypermentalizing, on the other hand, can produce feelings of emptiness as the interpretations of mental states are not sufficiently rooted in experienced life conditions. Both the loss of influence and feelings of emptiness might be elements in the process generating a depressive condition (Luyten, Fonagy, et al., 2019).

Despite the theoretically close relation between mentalizing and depressive conditions, very few studies have explored the role of mentalizing impairments in depression, and even fewer studies have investigated the potential role of mentalizing as a mechanism of change in psychodynamic therapy. Moreover, the few existing studies in this area have found inconsistent results (Fischer-Kern \& Tmej, 2019; Katznelson, 2014). Whereas some studies did not find mentalizing impairments in patients with depression (Taubner et al., 2011), others reported that patients with depression had significantly lower levels of mentalizing compared with normal controls (e.g., Ekeblad et al., 2016; Fischer-Kern et al., 2013). In this context, it has been suggested that impairments in mentalizing are typically observed in patients with more severe and/or chronic depression (Fischer-Kern \& Tmej, 2019; Katznelson, 2014). Fischer-Kern et al. (2013), for instance, reported a positive correlation between the number of depressive episodes and impairments in mentalizing. Studies investigating the impact of mentalizing on treatment outcome also have yielded inconsistent results. Taubner et al. (2011), for instance, did not find pretreatment RF to predict outcome. In contrast, a processoutcome study by Ekeblad et al. (2016) reported that higher initial RF was related to more symptom reduction over the course of therapy. Given the paucity of research on mentalizing and depression and the inconsistent conclusions, more studies are needed.

Particularly informative would be studies that assess changes in mentalizing and depressive symptoms over the course of therapy. Such studies would add to knowledge about mentalizing both as a prognostic factor and as a mechanism of change. Furthermore, the combination of these perspectives would elucidate the importance of mentalizing as a stable skill and a fluctuating resource. Mentalizing as a product of lifelong development processes is expected to be a traitlike capacity requiring time and effort to change (Fonagy et al., 2002; Holmes, 2009). On the other hand, mentalizing is expected to fluctuate within the range of the individually developed capacity due to situational and emotional context (Jurist, 2018; Luyten, Fonagy, et al., 2019). As described earlier, mentalizing can be expected to fluctuate due to arousal and narrow-mindedness related to a depressive condition. And, in addition, the patient's level of developed and more firmly defined mentalizing skills can be related to his or her potential to profit from therapy or not. Both perspectives can provide important knowledge about the relation between mentalizing and depression.

Studies including frequent measures over the course of therapy make the assessment of the mentalizing construct a key issue. The most often used operationalization of mentalization has involved assessing RF. Initially, RF was assessed by using extensive interviews concerning relational experiences and relational reflection in general (Jurist, 2018). The interviews used for this purpose are the Adult Attachment Interview (AAI; Hesse, 2016) and the Parent Development Interview (PDI; Slade et al., 2004). As interviews are a costly and time-consuming method, research relying on interviews used small samples and infrequent measures (Katznelson, 2014; Luyten, Malcorps, et al., 2019), highlighting the need for an RF questionnaire. The studies of mentalizing already reviewed applied an interview to assess RF in samples ranging from 20 to 86 depressed participants. Fischer-Kern et al. (2013) measured RF of each patient once, while Ekeblad and colleagues and Taubner and colleagues measure RF three times over the course of therapy (baseline, midsession, and a defined end session). The use of interviews particularly limits the frequency of measures over the course of a treatment process. In recent years, several well-validated questionnaires have been developed for this purpose (Luyten, Malcorps, et al., 2019). Compared to interviews, a questionnaire expands the possibility to explore the processes that unfold in therapy by the use of repeated and frequent measurements.

Repeated measurements reveal how various processes unfold over time and enable the study of reciprocal relationships among variables (Johnson et al., 2018). Thus, variations within a person over time may be disaggregated from the individual mean level, and in this way separating the within-person and between-person effects (Curran \& Bauer, 2011; Wang \& Maxwell, 2015). In the presence of systematic change over time, linear or nonlinear trends may be included at the between-person level, while within-person effect then is the additional time-specific deviations from these trends (Curran et al., 2014).

The extent to which within-patient time-specific fluctuations in the mentalizing function affect fluctuations in depression allows for 
an investigation of the mechanisms of change (Kazdin, 2007), however, without giving final evidence of causal effects (Mund \& Nestler, 2019). Furthermore, differences in trends between persons may disclose what characterize persons who profit or do not profit from treatment and if mentalizing can be a plausible explanation for the observed changes. The presence of mentalizing skills might be a factor contributing to moderately depressed individuals' ability to benefit from treatment, while poor or absent mentalizing capacity might be part of the dynamics underlying treatment resistance in individuals with severe depression. A statelike component reflects changes occurring over the course of therapy at the within-person level (Zilcha-Mano, 2017), whereas a trait-like component reflects differences at the between-person level. Thus, testing within-person effects and testing trend relations at the between-person level should be relevant in treatment research. Knowledge about patient characteristics related to outcome and knowledge about mechanisms of change are both needed in the development of therapeutic treatments. No studies investigating RF have studied both within- and between-patient effects on change in depression and mentalizing.

The aim of the present study was therefore to explore changes in patients' RF and their depressive symptoms using data from a naturalistic outcome study of intensive psychodynamic inpatient therapy for patients with depression. The following four hypotheses were tested. First, we expected that patients' symptoms of depression would be found to decrease both over the course of treatment and in the follow-up period. Second, the patients' RF was expected to improve over the course of treatment and during the follow-up period. Third, at the between-person level, patients with higher initial level of RF would also show greater reduction in depression in the treatment period. Fourth, at the within-person level, a patient who experiences a higher increase than expected in RF would experience a subsequent larger reduction in depression than expected.

\section{Method}

\section{Participants}

Participants were recruited among patients admitted to the VITA (latin for life) unit at Modum Bad Psychiatric Center in Norway. Modum Bad is a specialist hospital and the VITA-treatment program has been developed to address the needs of patients who have mental health problems and who identify existential and/or religious struggles as part of their problems (Stålsett, 2012). Depression was by far the most prevalent disorder in this patient population. In the current sample, $96 \%$ were diagnosed with depressive disorders, while $4 \%$ were diagnosed with moderate bipolar disorder. Furthermore, there was also a high level of comorbidity with panic disorder $(23.2 \%)$ and social phobia (17.8\%) as the most prevalent comorbid disorders. See Table 1 for more sample characteristics. For most patients, local psychiatric treatment had been tried without a satisfactory outcome before referral to Modum Bad, although in a minority of cases (in this sample $8.9 \%$ ), the patient was referred directly to Modum Bad and the VITA unit due to their expressed need to include religious and existential issues in therapy. Furthermore, the average duration of the depressive condition was 11.7 years for the patients participating in the study. Combined with nonsatisfactory results from former treatments, this signals a considerable treatment resistance.
Table 1

Sample Characteristics at Pretreatment $(\mathrm{N}=56)$

\begin{tabular}{|c|c|}
\hline Characteristics & Mean $(S D)$ \\
\hline \multicolumn{2}{|l|}{ Demographic } \\
\hline Age & $46.2(11$ \\
\hline Duration of depression $^{\mathrm{a}}$ & $11.7(10.3)$ \\
\hline Female/male $(n)$ & $38 / 18$ \\
\hline Married/live alone/other ${ }^{\mathrm{b}}(n)$ & $28 / 25$ \\
\hline \multicolumn{2}{|l|}{ Education } \\
\hline \multicolumn{2}{|l|}{ Upper secondary } \\
\hline \multicolumn{2}{|l|}{ College } \\
\hline \multicolumn{2}{|l|}{ University } \\
\hline \multicolumn{2}{|l|}{ Other ${ }^{\mathrm{c}}$} \\
\hline \multicolumn{2}{|l|}{ Work last 6 months $(n)$} \\
\hline \multicolumn{2}{|l|}{ Disabled $^{\mathrm{d}}$} \\
\hline \multicolumn{2}{|l|}{ Unemployed } \\
\hline \multicolumn{2}{|l|}{ Sick leave } \\
\hline \multicolumn{2}{|l|}{ Partly employed } \\
\hline \multicolumn{2}{|l|}{ Employed } \\
\hline \multicolumn{2}{|l|}{ Treatment } \\
\hline \multicolumn{2}{|l|}{ Former treatment } \\
\hline \multicolumn{2}{|l|}{ No former treatment } \\
\hline \multicolumn{2}{|l|}{ Outpatient psychiatric treatment } \\
\hline \multicolumn{2}{|l|}{ Inpatient psychiatric treatment } \\
\hline Outpatient and inpatient psychiatric treatment & \\
\hline Antidepressant medication before treatment $\mathrm{e}^{\mathrm{e}}$ & $21 /$ \\
\hline
\end{tabular}

Note. ${ }^{a}$ Duration of depressive condition is reported in years. For five participants, the duration of the depressive condition is unknown.

${ }^{\mathrm{b}}$ Other includes widows/widowers.

${ }^{\mathrm{c}}$ Other includes practically oriented education and courses not arranged by the university.

${ }^{\mathrm{d}}$ Disabled indicates participants who received state benefit payments due to illness.

e Twenty-one patients used antidepressant medication before treatment. Thirty-four patients did not.

The sample consisted of $67 \%$ women. The average age was 46 years and 59\% had completed college education. See Table 2 for an overview of comorbidity.

All participants were assessed for treatment based on a 2-day pretreatment evaluation. The pretreatment evaluation comprised an

Table 2

Diagnoses at Pretreatment in the Total Sample $(\mathrm{N}=56)$

\begin{tabular}{lrr}
\hline \multicolumn{1}{c}{ Characteristics } & $N$ & $\%$ \\
\hline Average number of diagnosis/patient (mean) & 1.92 & \\
Major depressive disorder & 26 & 46.4 \\
Major depressive episode & 5 & 8.9 \\
Moderate depressive episode & 5 & 8.9 \\
Dysthymia & 18 & 32.1 \\
Bipolar disorder, moderate & 2 & 3.6 \\
PTSD & 1 & 1.8 \\
PD/A & 13 & 23.2 \\
SAD & 10 & 17.8 \\
OCD & 5 & 8.9 \\
GAD & 7 & 12.5 \\
SF & 3 & 5.4 \\
Alcohol abuse & 1 & 1.8 \\
Alcohol dependence & 3 & 5.4 \\
PD Cluster C & 8 & 14.3 \\
\end{tabular}

Note. $\mathrm{PTSD}=$ Posttraumatic Stress Disorder; $\mathrm{PD} / \mathrm{A}=$ Panic Disorder with and without Agoraphobia; SAD $=$ Social Phobia; $\mathrm{OCD}=$ ObsessiveCompulsive Disorder; GAD = Generalized Anxiety Disorder; PD = Personality Disorder. 
extensive clinical interview and two semistructured clinical interviews, the Mini-International Neuropsychiatric Interview (MINI; Lecrubier et al., 1997) for Axis I diagnoses, and the Structured clinical interview for Diagnostic and Statistical Manual of Mental Disorders (DSM-IV) Axis II diagnoses (SCID-II; First et al., 1997).

To be eligible for the present study, participants had to meet the criteria for major depressive disorder, depressive episode, moderate bipolar disorder, or dysthymia as assessed by the MINI and as indicated by scores on the Beck Depression Interview (BDI-II; Beck et al., 1996; cutoff > 14) and the Symptom Checklist-90 (Derogatis \& Savitz, 1999; cutoff > 1.0). Exclusion criteria were as follows: (a) Symptoms or diagnosis of severe bipolar affective disorder; (b) symptoms or diagnosis of severe cluster B personality disorder; (c) recent or current suicide risk; (d) recent psychotic symptoms or a diagnosis of psychotic disorder; and/or (e) active substance abuse. Assessment of the exclusion criteria was integrated in the application of the MINI. Substance abuse was assessed using the self-reports of Alcohol Use Disorders Identification Test (AUDIT; Saunders et al., 1993) and Drug Use Disorders Identification Test (DUDIT; Berman et al., 2007). During pretreatment assessment, patients were also instructed to terminate or reduce the use of psychotropic medication. At treatment start, 21 patients applied some psychotropic medication. The changes in depressive symptoms over the course of treatment were related to psychotropic medication and no such associations were found. The study was approved by the Norwegian regional ethical committee (2011/197/REK Southeast).
A total of 57 patients were initially included in the study during the data collection period between March 2011 and November 2012. The VITA unit consisted of two treatment teams which were responsible for one group of patients each. A group counted seven or eight patients. The data were collected from 10 groups comprising 78 patients ( 2 groups of 7 patients and 8 groups of 8 patients). Recruitment to the study was conducted by applying the clinical criteria for treatment used at the unit. Furthermore, the admitted patients were informed about the study and invited to participate. Of note, 63 accepted, and 15 declined due to the anticipated workload connected to participation. Due to limited staff in the research project, we could not include all available patients in the study. Accordingly, six patients who had conceded to participate could not be included. When we had to exclude participants, men were preferred in the study as they generally were in minority in the population of patients referred to the unit. In summary, this adds up to the 57 patients who were included in the study. See Figure 1 for the flowchart of study enrollment.

\section{Treatments}

The VITA treatment model is designed as a 3-month inpatient treatment and includes individual and group sessions. Each individual treatment group comprised seven or eight patients. The VITA treatment model consists of four building blocks: (a) object relations theory, (b) psychodynamic existential psychology, (c) narrative

Figure 1

Consolidated Standards of Reporting Trails (CONSORT) Diagram of Patient Flow

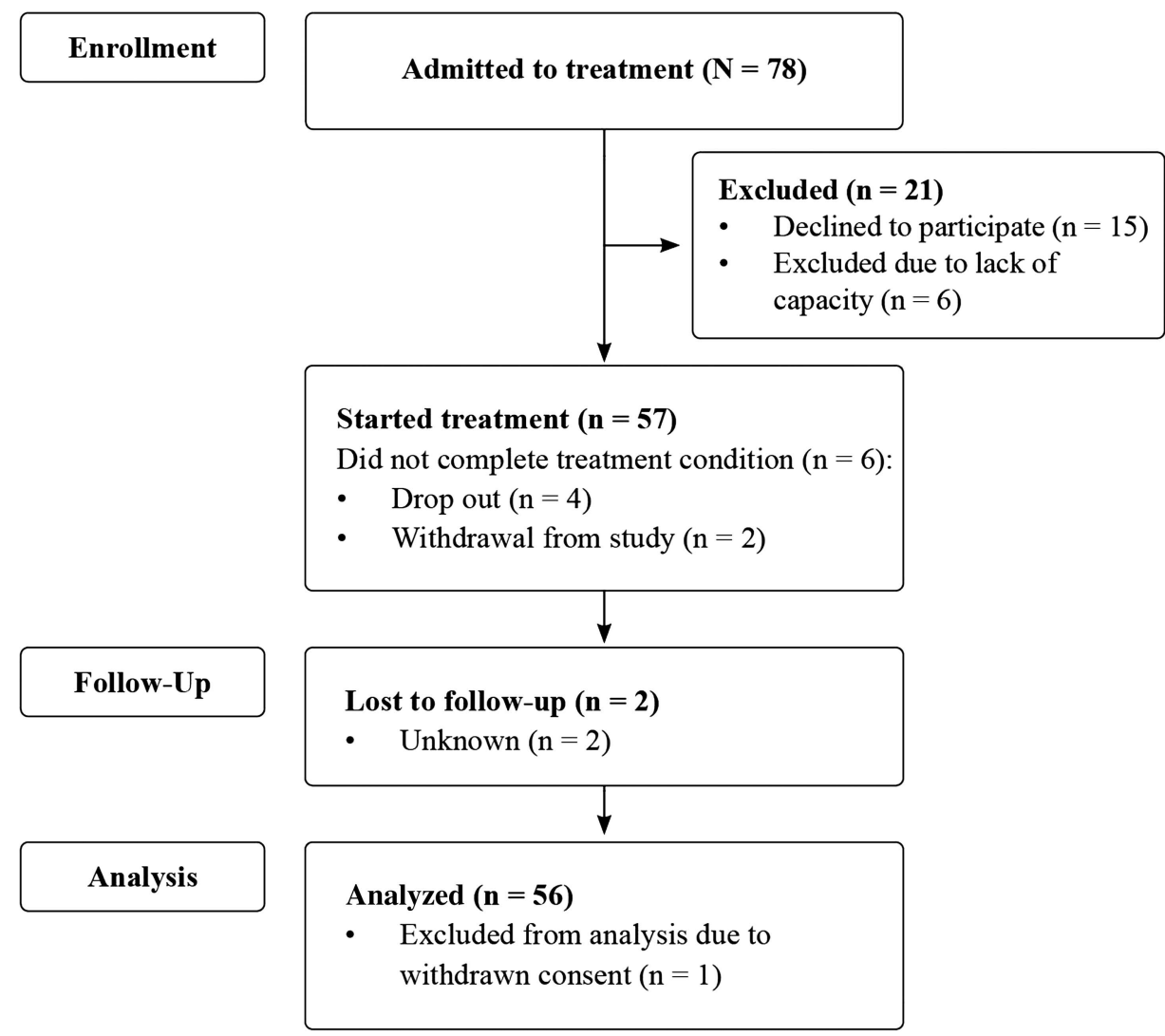


theory, and (d) affect theory (Stålsett, 2012). The treatment strategies are closely related to the chosen theoretical perspectives. Accordingly, the following treatment strategies are included within the frames of the VITA treatment model: (a) Identify existential and religious issues and related painful feelings; (b) explore life stories and rigid conclusions; (c) identify origins of emotional conflicts and relational patterns in early-life relational experiences; and (d) intensive focus on specific affects. The treatment strategies aim to improve metareflection, mentalizing, and mindfulness (Rizzuto et al., 2017; Stålsett, 2012).

The overarching integrative functions of the VITA treatment model are suited for processes that might elicit improved mentalizing. This is emphasized by two important perspectives in the treatment model. First, the integration of a multitude of perspectives helps the patient to experience the changes in perceptions, understanding, and feelings that result when viewing situations and relations from different angles. The ability to integrate emotions, relations, reflection, and metacognitions into a nuanced awareness of one's own and other individuals' intentions can be understood as a basic definition of the mentalizing process (Allen et al., 2008). In addition, this approach can be expected to produce more acceptance for the opacity of mental processes. Second, the patients are invited to take part in a culture of inquiry. The idea of a culture of inquiry is a recognition of the need to investigate the content of mental processes. For patients who hypomentalize excessively, the recognition and expression of their basic perceptions are the first steps in the process of identifying the difference between mental representations and the outside world. Patients who mainly hypermentalize will profit from exploring the genuine reactions and emotions instead of just producing a verbal flow of pseudo mentalizing.

Though the VITA treatment model does not apply the manualized techniques for mentalizing-based therapy, the processes initiated as the treatment elements interact can be expected to help the individual patient develop new or improved mentalizing capacity. Although resting on object relational therapy as a cornerstone of treatment, the model also includes additional elements that interact in the therapy process. The systematic inclusion of the God relation as an object relation and the identification of existential issues and related painful feelings are elements in the treatment model that might reduce generalizability to other psychodynamic inpatient treatments.

Two treatment teams provided the treatment, with each team comprising two or three therapists, two psychiatric nurses, and one assistant. The teams were trained in the VITA treatment model through internal courses given by the developers and by supervision from a group analyst who had supervised the VITA-treatment teams from the start of the program in 1998. The project leader was individually supervised by the psychiatrist who contributed substantially to the development of the treatment model and who is also vastly experienced in the clinical application of the treatment model.

\section{Adherence}

To strengthen adherence to the VITA treatment model, the two teams discussed the main elements in the model until consensus on principles for clinical practice was obtained (Stålsett, 2012). Audio recordings of the four categories of group sessions were made in the first session, in one session during the treatment period, and in the last session to assess adherence in the six last groups of participants, leading to a total of 56 audiotapes. A random selection of 30 audiotapes was examined by an experienced psychiatric nurse who had worked in the VITA unit for approximately 10 years. The examination was conducted by the use of a questionnaire consisting of five questions with a 5-point Likert scale. The questionnaire was developed by the first author. Twenty of the 30 tested audiotapes were reexamined by an experienced VITA therapist to measure interrater reliability. Before starting the adherence assessment, the psychiatric nurse and the VITA therapist were trained in assessment principles through two sessions with the first author. The agreement between the raters on the adherence scores was 95\%, and the interrater agreement on the adherence ratings using a one-way random intraclass correlation $[\operatorname{ICC}(1,1)]$ was .79 with an average measure, which is good to excellent.

\section{Therapists}

One of the seven different therapists was in charge of each participant's therapeutic process during the treatment period and also provided the individual therapy sessions for the given patient. Two of these therapists were psychiatrists, two were psychologists, two were ministers with extensive training in psychotherapy, and one was a social worker certified in individual psychotherapy and in group analysis.

\section{Measures}

Patients who consented to participate in the study completed depression and mentalization (RF) questionnaires. During treatment, the patients' depressive symptoms and their RF were measured every week by the BDI-II and the Reflective Functioning Questionnaire (RFQ; Fonagy et al., 2016). The BDI-II and RFQ were also completed at baseline and at treatment termination, and at 1-year follow-up.

The BDI-II (Beck et al., 1996) is an instrument with 21 items, measuring the symptoms of depression in the past week. The items are rated on a Likert scale from 0 to 3 , and the total score ranges from 0 to 63 . The BDI-II has been found to be reliable and valid for measuring depressive symptoms (Sprinkle et al., 2002). At baseline in this study, Cronbach's alpha showed good internal consistency reliability $(\alpha=.86)$. Furthermore, the Norwegian translation of the BDI-II has been found to have sufficiently good psychometric properties (Siqveland \& Kornør, 2011).

The RFQ is a brief questionnaire assessing certainty (RFQ-C) and uncertainty (RFQ-U) with regard to mental states (Fonagy et al., 2016). It comprises eight items, which are scored on a 7-point Likert-type scale, ranging from strongly disagree to strongly agree. When analyzing the RFQ, the raw scores are recoded into a 4-point scale from 0 to 3 producing a maximum score of 18 and a minimum score of 0 . This recoding is done to prevent the scores indicating hypermentalizing and hypomentalizing to conflate, and in addition to be able to discern RFQ-C and RFQ-U (Fonagy et al., 2016). Accordingly, moderate to high scores on the RFQ-C indicate adaptive certainty about mental states. On the other hand, low scores reflect a lack of certainty about mental states of both the self and others which denote hypomentalizing. Very high scores reflect excessive certainty about mental states and signal 
hypermentalizing. High scores on the RFQ-U subscale indicate severe problems with hypomentalizing that are typically observed in patients with different pathological conditions. In contrast, the RFQ-C assesses both adaptive and maladaptive features of mentalizing (De Meulemeester et al., 2018; Fonagy et al., 2016).

The internal consistency for both the RFQ-U and the RFQ-C is satisfactory (Cronbach's alphas of .77 and .65, respectively) and the test-retest reliability after 3 weeks was good $\left(r_{\mathrm{s}}=.84\right.$ and.75 for RFQ-U and RFQ-C, respectively) (Fonagy et al., 2016). RFQ was validated in a factor analysis starting with a 54-item questionnaire concerning theoretical and empirically described aspects of the four dimensions in the mentalizing construct [(a) automatic/controlled, (b) internally focused/externally focused, (c) self-oriented/otheroriented, and (d) cognitive/affective]. The factor analysis identified two dimensions in mentalizing (reflective certainty and reflective uncertainty) and from these the eight-item RFQ applied in this study was developed. Furthermore, the construct validity is strengthened by empirical support for discriminant validity (discriminated between patients and controls) and convergent validity (correlated with measures of empathy, mindfulness, and perspective taking; Fonagy et al., 2016).

The original English-language version of the RFQ was translated into Norwegian by researchers at the Department of Personality Psychiatry, Clinic for Mental Health and Addiction, Oslo University Hospital, using the translation-back translation method. The back translation was discussed and approved by Anthony Bateman, one of the developers of mentalization-based treatment. Cronbach's alpha of the total scale showed satisfactory reliability $(\alpha=.71)$. No tests have been carried out to provide evidence related to psychometric properties of the Norwegian version of RFQ.

\section{Statistical Analyses}

Descriptive analyses (mean, $S D$, and frequency) were computed with SPSS 24 (IBM Corp, 2017). Latent Growth Curve (LGC) modeling was used to estimate mean values, variances, and covariances between level and change in the variables (Bollen \& Curran, 2006; Muthén \& Curran, 1997; Wang \& Wang, 2012), and implemented with Mplus 8.2 (Muthén \& Muthén, 2018). Time (weeks) was centered at baseline, which gives models describing trajectories from baseline levels. In addition, time was centered at mean time to estimate the linear change over the treatment period. The time intervals between the 12 measurements during the treatment period were somewhat different among individuals with the mean time at post treatment to be 13.4 weeks after baseline with some individual variation $(S D=1.1)$. Therefore, time was treated as random and represented in the analyses by a time variable. This resulted in loss of standard absolute Structure Equation Modeling (SEM) fit measures (for example, Root Mean Square Approximation (RMSEA). Full Information Maximum Likelihood (FIML) estimation was used to use all available data $(N=56)$ under the missing at random assumption (Enders, 2010), with standard error corrections Maximum Likelihood Robust (MLR) robust for potential nonnormal distributions (Wang \& Wang, 2012). Model fit was evaluated based on the Akaike Information Criterion (AIC), the Bayesian Information Criterion (BIC), and Sample-adjusted BIC (SABIC), with lower values indicating better model fit (Heck \& Thomas, 2015). Different models were compared with the Wald test (Hox et al., 2018). Only results from the final models are presented.

Standard procedures for model testing were followed (Kline, 2016). First, unconditional models with linear change over the treatment period of 12 weeks were tested. The models were evaluated based on mean and individual trajectories, and the estimated distributions of the residuals. Then, quadratic, cubic, and other functions of time factors were added and evaluated based on differences in fit indices and the Wald test. A simpler model was kept if more complex functions did not provide improvement in model fit or resulted in estimation problems. Random slopes were allowed for estimation but were fixed if not found to be statistically significant. This is similar to the random intercept fixed slope model in the linear mixed-effect/multilevel model (Hox et al., 2018). Time-specific residuals were constrained to be equal (homoscedasticity) and compared with models estimating unique and different residuals (heteroscedasticity) and compared in model fit. The simpler more parsimonious model was used if not being statistically inferior, as this model has better statistical power and higher precision in the estimates. Then, long-term changes from the end of treatment to 1-year follow-up were analyzed in separate models, using a piecewise LGC approach, giving level and nonlinear change in the treatment phase in one piece and the follow-up period from posttreatment to 1-year measurements in the last piece. The multivariate LGC conditional models level and change in BDI-II scores were regressed on level and change in RFQ-C and RFQ-U, respectively.

Dependent on the research problem, predictors, outcomes, or both variables may be disaggregated or detrended (Wang \& Maxwell, 2015). However, Curran and Bauer (2011) discussed and showed in a simulation study that variables changing systematically over time will give biased estimates of the within effects if detrending is not used, as time-specific variance attributed to the trend is not removed from the within-person variance. The detrending model gives unbiased estimates. The models therefore included trends if empirically supported in the multivariate models, and were expanded with structured residual relations to analyze the within-person effects (LGC Model with Structured Residuals [LCGM-SR]; Curran et al., 2014).If no trends were supported in outcome and predictors, a disaggregated model was specified. A combination was also possible, with detrending in one variable and disaggregation in the other. LCGM-SR uses phantom variables to represent residuals not accounted for by the latent intercept and slope factors (level and change). These residuals were modeled with stability parameters between residuals over time (time-lagged effects) and cross-lagged relations. The trends represent between-patient effects. Models with free residual estimates over time and constrained invariant estimates were compared. The full model is shown in Figure 2. The models with time-specific residuals (heteroscedasticity model) resulted in some cross-lagged relations late in the treatment period; however, it also resulted in estimation problems and loss of all between-patient level relations. Thus, this heteroscedastic model was problematic, and is therefore not presented.

Potential clustering effects caused by patients being nested within treatment groups were explored. Clustering in unconditional model variance were found for treatment groups (average level): BDI: $\mathrm{ICC}=.13$; RFQ-C: ICC $=.08$; and RFQ-U: ICC $=.00$. After including the time variables into the models, no differences in change over treatment clusters were found, and the random time 


\section{Figure 2}

Illustration of Analyzed Models of Beck Depression Interview (BDI)-II and Reflective Functioning Questionnaire (RFQ) (Certainty and Uncertainty) During Treatment From Baseline Before Treatment and Posttreatment at About 12 Weeks

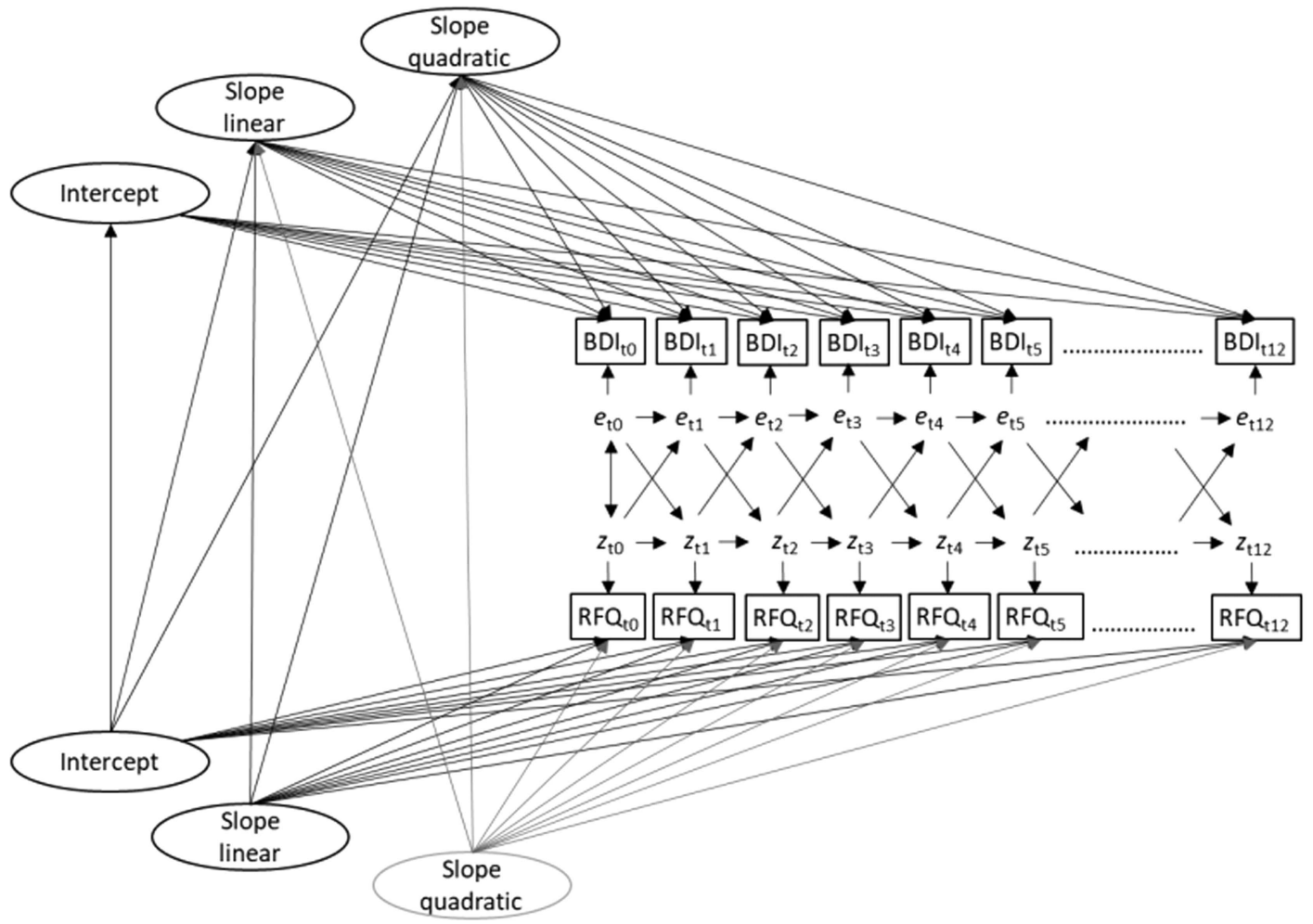

Note. Level and change (linear and quadratic) in BDI-II predicted by level and change in RFQ. Unconditional models tested for nonlinearity in change with quadratic and cubic slopes. Structured residuals with stability (autoregression) over time within each set of residuals and cross-lagged relations between BDI-II and RFQ residuals. See also the appendix "MPlus Analysis script for the Latent Growth Curve Model With Structured Residuals (LGCM-SR) for BDI-II and RFQ-C"

(slope) part of group clustering had to be fixed, indicating patients to be somewhat different over treatment groups when coming to treatment, but not changing differently over groups. Therefore, to keep precision and statistical power, group clustering was left out of the final models.

\section{Results}

The descriptive information of BDI and RFQ variables at baseline given in Table 3 shows some individual variations around the mean values and that all three variables showed relatively normal distributions. The ICC results show most stability in RFQ Certainty and less in Uncertainty scores.

\section{Hypothesis 1: Reduction in Depressive Symptoms}

A nonlinear change model, including the quadratic function, provided the best fit to model changes in depressive symptoms over the treatment period of 12 weeks and provided a significantly better fit than a linear model (Wald difference test $=12.09, d f=2$, $p=.002$ ) (see Table 4). As shown in Figure 3, symptoms of depression first increased during treatment, then leveled out (deceleration of the linear increase), and finally decreased to below baseline levels toward the end of treatment. The mean time-centered model showed the average linear reduction to be $\alpha=-.17, p=.047$, and reduction of .17 per week during the treatment phase. The intercept value at mean treatment time was found to be 18.28 , $p<.001$. The cubic or other nonlinear functions did not fit the observed data equally well and therefore were not presented. Model fit was best when estimating unequal residuals over time (Wald test $=40.38, d f=12, p<.001$ ). Moreover, variances in level, linear, and quadratic change were found to be statistically significant,

\section{Table 3}

Descriptive Information of Sum Scores at Baseline: Beck Depression Interview (BDI) and Mentalizing Certainty and Uncertainty $(\mathrm{N}=56)(I C C=$ Intraclass Correlation; $R F Q=$ Reflective Functioning Questionnaire)

\begin{tabular}{lrccr}
\hline \multicolumn{1}{c}{ Variable } & Mean & $S D$ & Skewness & ICC \\
\hline BDI & 15.94 & 6.96 & .26 & .60 \\
RFQ_Certainty & 3.13 & 2.13 & .55 & .62 \\
RFQ_Uncertainty & 4.79 & 1.95 & .88 & .51 \\
\hline
\end{tabular}


Figure 3

Estimated Beck Depression Interview (BDI)-II Scores Over Treatment at Mean and $\pm 1 / 4$ SD Level

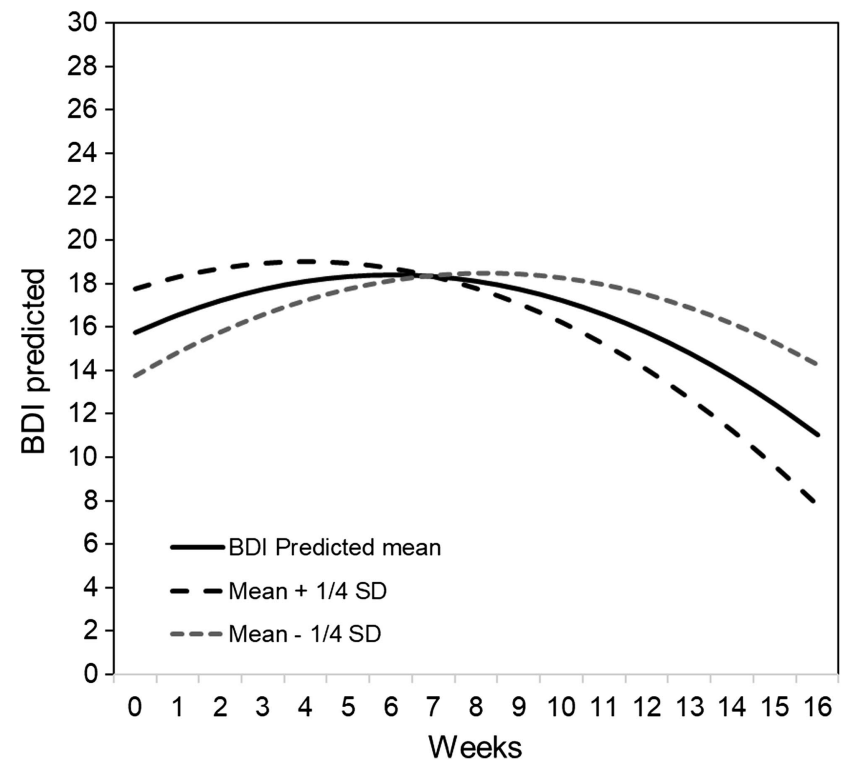

suggesting that patients differed in terms of the level and pattern of change during treatment (Figures 3 and 4). The results showed stronger linear increases among patients with lower baseline depression scores than among those who had higher levels of baseline depression. Furthermore, patients who experienced a stronger linear immediate increase in depressive symptoms also showed a stronger nonlinear reduction over time.

Including the change in BDI-II score from posttreatment to 1-year follow-up into the LGC model, there was a significant reduction $\left(\alpha_{\text {mean }}=-.07, p=.016\right)$, with some individual variation $\left(\sigma^{2}=.02\right.$, $p=.005)$. The effect size for this period was estimated to be $d=.56$ (estimate/SD of change), a moderate effect size (moderate effect size $.8>,<.2$; Clark-Carter, 1997). The effect size for the linear change during the total period of treatment and 1-year follow-up was $d=1.47\left(\alpha_{\text {mean }}=-.09\right.$ per week, $\left.p=.001\right)$, representing a very large effect size (large effect size $>.8$; Clark-Carter, 1997).

\section{Hypothesis 2: Improvement in RF}

Mean change in RFQ-C showed a nonlinear trajectory during treatment, deteriorating during the first period of treatment, then leveling out, and finally improving back to baseline levels toward the end of treatment (see Table 4 and Figure 5). At mean level, RFQ-U showed no statistically significant linear change over time, which implied no improvement. However, there was statistically significant variation in individual level and change during treatment for both variables, and relations between levels, linear, and nonlinear change. This means that some patients increased while others decreased in RFQ-U scores, and that the rate of change was dependent on the baseline level. Regarding changes from posttreatment to 1-year follow-up, there were no significant reductions in either RFQ-C or RFQ-U in the sample as a whole $\left(\alpha_{\text {mean }}=.01\right.$, 
Figure 4

Estimated Individual Beck Depression Interview (BDI)-II Scores Over Treatment

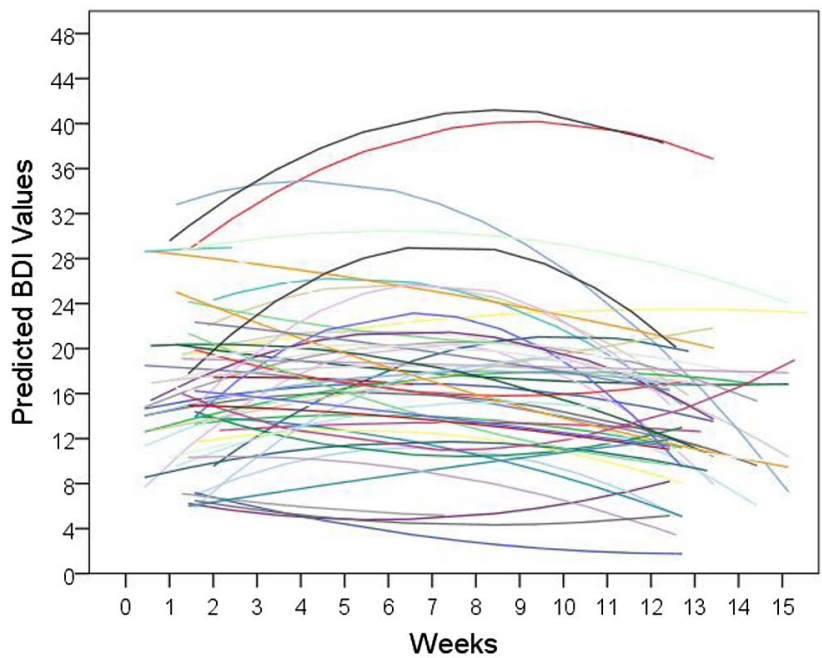

Note. See the online article for the color version of this figure.

$p=.072 ; \alpha_{\text {mean }}=-.01, p=.675$, respectively). No statistically significant variance in change was found either.

\section{Hypothesis 3: Initial Level of RF and Reduction in Depression}

Changes in depressive symptoms were predicted by the RFQ-C. Specifically, linear changes in BDI-II scores were significantly (or trend-significantly) related to the following on RFQ-C: baseline levels $(b=-.51, p=.022)$, linear changes $(b=-16.80, p=.043)$, and quadratic changes $(b=-312.03, p=.057)$. Furthermore, quadratic nonlinear changes in BDI-II scores were predicted

Figure 5

Estimated Mean Change in Reflective Functioning Questionnaire (RFQ)-C

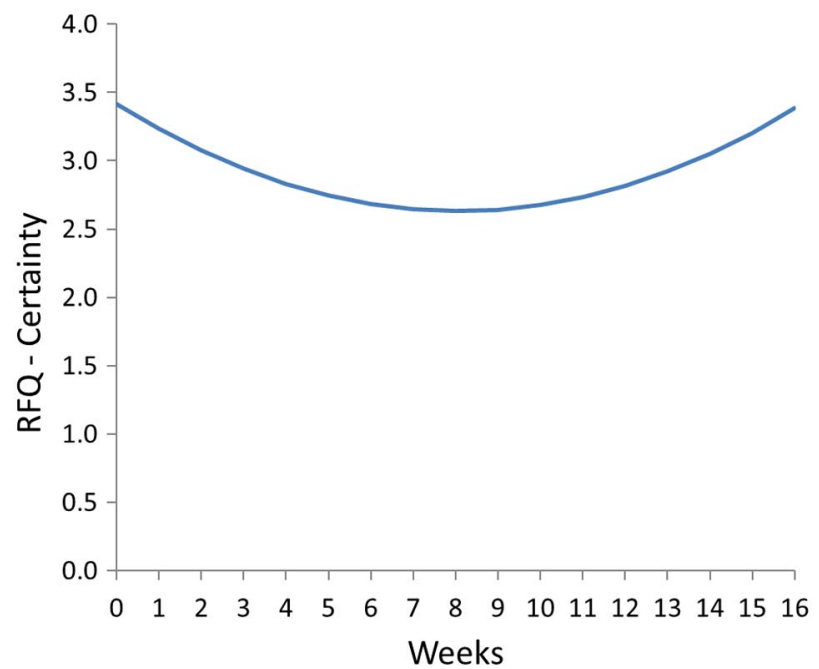

Note. See the online article for the color version of this figure. by baseline levels $(b=.03, p=.040)$, linear changes $(b=1.15$, $p=.048)$, and a trend for quadratic changes in RFQ-C $(b=21.71$, $p=.058)$. Baseline BDI-II levels were not related to baseline levels in RFQ-C $(b=.36, p=.581)$. Hence, patients with higher initial levels of RFQ-C showed less temporary increase in depressive symptoms at the start of treatment, and therefore, faster reduction in depressive symptoms over time. On the other hand, patients with lower levels of RFQ-C when starting treatment experienced stronger increase, but transient, in the observed levels of depressive symptoms. In addition, patients who demonstrated stronger temporary reductions in RFQ-C scores also experienced stronger temporary increases in BDI-II scores during the treatment period.

Regarding RFQ-U, baseline levels of RFQ-U did not predict either linear or quadratic change in BDI-II scores $(b=.03$, $p=.970 ; b=-.01, p=.937$, respectively). However, changes in BDI-II scores were related to linear changes in RFQ-U (BDI-II linear change: $b=66.65, p<.001$; quadratic change: $b=-4.66$, $p<.001)$. Together, these findings show that those patients reporting the greatest reductions in RFQ-U also reported the smallest temporary increase in BDI-II, and therefore reduced their symptom levels earlier on in the treatment phase.

\section{Hypothesis 4: Within-Person Effects of RF on Depression}

The LCGM-SR models showed no statistically significant crosslagged average associations between RFQ-C and BDI-II residual scores (RF-C $\rightarrow$ depression residuals after accounting for stability relations: $b=.12, p=.612$; depression $\rightarrow$ RF-C: $b=.01, p=.565$, residuals specified equal over time). For RFQ-U, similarly, there were no significant cross-lagged residual relations from RFQ-U to BDI-II $(b=.32, p=.149)$ or from BDI-II to RFQ-U $(b=.01$, $p=.753)$

\section{Discussion}

The present study aimed to investigate changes in depression and mentalizing over the course of 3 months of intensive inpatient psychodynamic therapy. The first hypothesis that patients' symptoms of depression would decrease over the course of treatment was confirmed. The results showed a statistically significant reduction in depressive symptoms, representing a very large effect size. Interestingly, on average, patients typically showed an increase in depressive symptoms in the first few weeks of the treatment, before improving. The temporary increase in depressive symptoms might have been due to the activation of painful emotions during the initial phase in therapy (Luyten, Fonagy, et al., 2019; Owen et al., 2015). However, the results showed individual differences in the patterns of change. Some patients experienced a worsening of symptoms before recovering, whereas others experienced a gradual linear decrease in symptoms.

The reduction in depressive symptoms continued from posttreatment to follow-up. The finding that treatment effects were maintained in the longer term is consistent with other studies of psychodynamic therapy for depression (Driessen et al., 2015). As these were patients who had had several unsuccessful treatments in the past, it is very unlikely that patients would show spontaneous recovery (Rost et al., 2019), although the naturalistic design of the study limits our ability to attribute the observed effects to the treatment as such. Moreover, as we have no data on additional 
treatment seeking, the observed effects might also have been due to additional treatment.

Our second hypothesis - that over the course of treatment patients' RF would improve-was not supported. In the total sample, there were no significant increases in certainty or decrease in uncertainty of mentalizing. However, there were statistically significant individual variations in the level of change in certainty and uncertainty. At mean level, RFQ-C fell to a significantly lower level compared with baseline in the initial phase of treatment and then increased again to the baseline level in the later phases of treatment. The initial increase in depression might have contributed to this deterioration of mentalizing, as it has been a well-validated finding that arousal typically impairs controlled mentalizing (Luyten, Fonagy, et al., 2019). The lack of improvement at group level in mentalizing capacity over the course of therapy may also be related to the relatively brief treatment format. Yet, individual differences in trajectories of change show that some individuals improved while others did not. Reasons for such differences can be further analyzed in later studies.

Mentalizing can be understood both as a stable trait-like capacity and a fluctuating state-like component. In psychodynamic comprehension, the patient's trait-like mentalizing capacity is the general ability to mentalize, which does not address how mentalizing changes over the course of therapy (i.e., state like; Zilcha-Mano, 2017). The within-patient change, on the other hand, can be considered a state-like component that reflects changes in mentalizing capacity occurring over the course of therapy (Zilcha-Mano, 2017). When finding systematic trends in the present study, changes within patients are separated at both within and between levels as within-person residuals and between-person slope factors (Curran \& Bauer, 2011). This decomposes states as linear or nonlinear trends and fluctuations around these, while traits represented by the intercept factor may be defined as a stable level during the study (Mund \& Nestler, 2019). This brings us to our third and fourth hypothesis, concerning the association between RF and changes in depression.

At the between-person level, levels in RF, and certainty about mental states in particular, were related to changes in depressive symptoms. Thus, the third hypothesis was confirmed. The results show how some individuals were at high levels at baseline, while others improved during treatment. It is interesting to note that patients who reported the highest level of improvement in certainty/uncertainty also had a smaller temporary increase and then a greater reduction in BDI-II score. This finding parallels Ekeblad et al.'s (2016) finding that better capacity for mentalizing at baseline was related to greater reductions in symptoms over the course of therapy. It also corresponds to Fonagy et al.'s (2016) finding that RFQ-C is inversely related to depressive symptoms. These results can be interpreted as an indication that individuals with higher levels of mentalizing are protected and more robust when faced with the emotional strain associated with the initial phase of therapy. Furthermore, this finding is in line with Rost et al.'s (2019) finding that more severe and treatment-resistant depressive conditions are related to more maladaptive states of submissive or dismissive relations to self and/or others, which can be expected to be related to impairments in RFQ-C and RFQ-U as well.

This can be understood as expressions of protective capacities developing from mentalizing skills and as expressions of vulnerability resulting from the lack of mentalizing skills. Accordingly, the patient's pretreatment level of mentalizing capacity provides important knowledge about the outcome that can be expected from therapy, suggesting three considerations. First, some patients might have a sufficiently high initial level of mentalizing capacity to profit from the therapeutic processes initiated by the elements of an intensive psychodynamic therapy, such as the VITA treatment model. Second, some patients might not have the level of mentalizing skills needed to benefit from treatment from at the start, but as the treatment process help them improve their mentalizing, they can engage in the therapeutic work in a more fruitful manner. This is supported by the negative correlation between baseline level and change, showing patients low on RF increasing more than those high on RF. Third, there might be another subgroup that do not have and never reaches a sufficient level of mentalizing to engage in a productive therapy process. For the RFQ-C scale, this can further be related to the level and change of depressive symptoms. Accordingly, the initial level and change in RFQ-C predict the level and change in depressive symptoms over the course of therapy. This indicates that the levels of mentalizing skills as measured by RFQ-C function both to protect some patients from aggravated depressive conditions and to facilitate these patients' benefit from therapy. In addition, this also identifies severity of depressive symptoms and low levels of mentalizing skills (as measured by RFQ-C) as possible predictive factors for treatment resistant depressive conditions.

Our fourth hypothesis was not supported as time-specific residual changes in RF did not predict later time-specific residual changes in depression not accounted for by the longitudinal trends. Accordingly, mentalizing was not corroborated as a general mechanism of change. There could be different reasons for not finding withinperson effects in the current study. First, there are statistical reasons associated with sample size, loss of residual variance in a model with trend functions, and 1-week lags. However, it should be noted that additional models with increasingly longer time lags, and models without the trends, did not alter these findings (results not presented). Second, mentalizing is a complex construct, the functions of which are far from fully mapped, and accordingly, we found it reasonable to explore its capacity as a mechanism of change in a therapy process. As exhibited by our results, no within-person effects were found and accordingly mentalizing cannot be considered a mechanism of change behind the temporarily reduction in depressive symptoms. Hence, further research is needed to uncover these processes.

The ability to access, contain, integrate, and express affects can be a separate object of future explorations due to its theoretical and empirical connection to depressive conditions (Diener et al., 2007) and its central role in the VITA treatment model (Stålsett, 2012). Future studies should also investigate possible effects related to specific aspects of the mentalizing construct, for instance, the capacity to mentalize affects (Jurist, 2018; Luyten, Malcorps, et al., 2019) or the external-internal dimension (Luyten, Malcorps, et al., 2019).

This study had several limitations. First, the use of self-report questionnaires to measure depression as well as RF may have influenced participants' responses and introduced self-report biases. As the assessment of mentalizing in particular implies the capacity for self-reflection, further studies using semistructured interviews and/or experimental tasks to assess mentalizing are needed. Second, as we have no information on additional treatment seeking, several unknown factors may explain the sustained effects observed in the current study. Third, because we had no control group, the treatment 
results of the depressed patients in our study cannot be compared to patients receiving other forms of treatment. This limits our possibilities to be conclusive about the cause(s) behind our treatment results. As both the sample and the treatment model have distinct features, the generalizability of our findings is also restrained. Further research with control groups and randomization to different therapy conditions will be conductive to explore the generalizability of our results. Fourth, the sample size represents another limitation, with estimation consequences regarding precision and statistical power.

Our study also has an important strength as it is the first study to disaggregate within- and between-person changes in RF and depressive symptoms over the course of therapy. As the VITA treatment model is associated with a statistically significant reduction in depressive symptoms both post therapy and at 1-year follow-up, it is of importance to seek the mechanisms of change behind these results. This study did not support that improved capacity to mentalize is a general mechanism of change behind the reduction in depressive symptoms at within-person level. Still, mentalizing skills are found to be a necessary component for many patients' therapy processes to be beneficial. Hence, the potential to represent group mean and individual differences in level and change as between-person effects and time-specific fluctuations within-person effects have provided nuanced knowledge about the mentalizing capacity's role in the treatment process. This is a strength related to our study. Future studies including larger numbers of participants may generate more robust results and also have the potential to identify subgroups in the population at baseline and compare their trajectories of change over the course of therapy, which could contribute important gains to the body of knowledge about the mechanisms of change in the VITA treatment model.

In conclusion, our results have illuminated some of the relations between depression and the capacity to mentalize. Mentalizing was not a mechanism of change. However, our results indicate that mentalizing is related to depression both in a positive, protective sense and in a negative, oppressive sense. The presence of mentalizing skills might be a factor contributing to moderately depressed individuals' ability to benefit from treatment, while poor or absent mentalizing capacity might be part of the dynamics underlying treatment resistance in individuals with severe depression.

\section{References}

Allen, J. G., Fonagy, P., \& Bateman, A. W. (2008). Mentalizing in clinical practice. American Psychiatric Accociation.

Bateman, A., \& Fonagy, P. (2015). Borderline personality disorder and mood disorders: Mentalizing as a framework for integrated treatment. Journal of Clinical Psychology, 71(8), 792-804. https://doi.org/10.1002/jclp.22206

Beck, A. T., Rush, A. J., Shaw, B. F., \& Emery, G. (1979). Cognitive therapy of depression. Guilford Press.

Beck, A. T., Steer, R. A., \& Brown, G. K. (1996). BDI-II manual. Psychological Corporation.

Berman, A. H., Palmstierna, T., Källmén, H., \& Bergman, H. (2007). The self-report drug use disorders identification test—extended (DUDIT-E): Reliability, validity, and motivational index. Journal of Substance Abuse Treatment, 32(4), 357-369. https://doi.org/10.1016/j.jsat.2006.10.001

Blatt, S. J. (1974). Levels of object representation in anaclitic and introjective depression. The Psychoanalytic Study of the Child, 29(1), 107-157. https://doi.org/10.1080/00797308.1974.11822616
Blatt, S. J. (2004). Experiences of depression: Theoretical, clinical, and research perspectives. American Psychological Association. https:// doi.org/10.1037/10749-000

Bollen, K. A., \& Curran, P. J. (2006). Latent curve models: A structural equation perspective. Wiley.

Clark-Carter, D. (1997). Doing quantitative psychological research: From design to report. Psychology Press.

Curran, P. J., \& Bauer, D. J. (2011). The disaggregation of within-person and between-person effects in longitudinal models of change. Annual Review of Psychology, 62, 583-619. https://doi.org/10.1146/annurev psych.093008.100356

Curran, P. J., Howard, A. L., Bainter, S. A., Lane, S. T., \& McGinley, J. S. (2014). The separation of between-person and within-person components of individual change over time: A latent curve model with structured residuals. Journal of Consulting and Clinical Psychology, 82(5), 879-894. https://doi.org/10.1037/a0035297

De Meulemeester, C., Vansteelandt, K., Luyten, P., \& Lowyck, B. (2018) Mentalizing as a mechanism of change in the treatment of patients with borderline personality disorder: A parallel process growth modeling approach. Personality Disorders, 9(1), 22-29. https://doi.org/10.1037/ per0000256

Derogatis, L. R., \& Savitz, K. L. (1999). The SCL-90-R, brief symptom inventory, and matching clinical rating scales. In M. E. Maruish (Ed.), The use of psychological testing for treatment planning and outcome assessment (pp. 679-724). Lawrence and Erlbamum Publisheres.

Diener, M. J., Hilsenroth, M. J., \& Weinberger, J. (2007). Therapist affect focus and patient outcomes in psychodynamic psychotherapy: A metaanalysis. The American Journal of Psychiatry, 164(6), 936-941. https:// doi.org/10.1176/ajp.2007.164.6.936

Driessen, E., Hegelmaier, L. M., Abbass, A. A., Barber, J. P., Dekker, J. J., Van, H. L., \& Cuijpers, P. (2015). The efficacy of short-term psychodynamic psychotherapy for depression: A meta-analysis update. Clinical Psychology Review, 42, 1-15. https://doi.org/10.1016/j.cpr 2015.07.004

Ekeblad, A., Falkenström, F., \& Holmqvist, R. (2016). Reflective functioning as predictor of working alliance and outcome in the treatment of depression. Journal of Consulting and Clinical Psychology, 84(1), 67-78. https://doi.org/10.1037/ccp0000055

Enders, C. K. (2010). Applied missing data analysis. Guilford Press.

First, M. B., Gibbon, M., Spitzer, R. L., Benjamin, L. S., \& Williams, J. B (1997). Structured clinical interview for DSM-IV axis II personality disorders: SCID-II. American Psychiatric Pub.

Fischer-Kern, M., Fonagy, P., Kapusta, N. D., Luyten, P., Boss, S., Naderer, A., \& Leithner, K. (2013). Mentalizing in female inpatients with major depressive disorder. Journal of Nervous and Mental Disease, 201(3), 202-207. https://doi.org/10.1097/NMD.0b013e3182845c0a

Fischer-Kern, M., \& Tmej, A. (2019). Mentalization and depression: Theoretical concepts, treatment approaches and empirical studies-an overview. Zeitschrift für Psychosomatische Medizin und Psychotherapie, 65(2), 162-177. https://doi.org/10.13109/zptm.2019.65.2.162

Fonagy, P., Gergely, G., Jurist, E., \& Target, M. (2002). Affect regulation, mentalization, and the development of the self. Other Press.

Fonagy, P., Luyten, P., Moulton-Perkins, A., Lee, Y. W., Warren, F., Howard, S., Ghiani, R., Fearon, P., \& Lowyck, B. (2016). Development and validation of a self-report measure of mentalizing: The reflective functioning questionnaire. PLOS ONE, 11(7), Article e0158678. https:// doi.org/10.1371/journal.pone.0158678

Heck, R. H., \& Thomas, S. L. (2015). An introduction to multilevel modeling techniques-MLM and SEM approaches using Mplus (3rd ed.). Taylor and Francis Group. https://doi.org/10.4324/9781315746494

Hesse, E. (2016). The adult attachment interview: Protocol, method and analysis and selected empirical studies: 1985-2015. In J. A. Cassidy \& P. R. Shaver (Eds.), Handbook of attachment: Theory, research, and clinical applications. Guilford Press. 
Holmes, J. (2009). Exploring in security: Towards an attachment-informed psychoanalytic psychotherapy. Routledge. https://doi.org/10.4324/ 9780203856321

Hox, J. J., Moerebeek, M., \& van de Schoot, R. (2018). Multilevel analysis. Techniques and applications (3rd ed.). Routledge. https://doi.org/10.4324/ 9781315650982

IBM Corp. (2017). IBM SPSS statistics for windows (Version 24.0).

Johnson, S., Hoffart, A., Nordahl, H., Ulvenes, P., Vrabel, K., \& Wampold, B. (2018). Metacognition and cognition in inpatient MCT and CBT for comorbid anxiety disorders: A study of within-person effects. Journal of Counseling Psychology, 65(1), 86-97. https://doi.org/10.1037/ cou0000226

Jurist, E. (2018). Minding emotions: Cultivating mentalization in psychotherapy. Guilford Press.

Katznelson, H. (2014). Reflective functioning: A review. Clinical Psychology Review, 34(2), 107-117. https://doi.org/10.1016/j.cpr.2013.12.003

Kazdin, A. E. (2007). Mediators and mechanisms of change in psychotherapy research. Annual Review of Clinical Psychology, 3, 1-27. https:// doi.org/10.1146/annurev.clinpsy.3.022806.091432

Kline, R. B. (2016). Principles and practice of structural equation modeling (4th ed.). Guilford Press.

Lecrubier, Y., Sheehan, D., Weiller, E., Amorim, P., Bonora, I., Sheehan, K., Janavs, J., \& Dunbar, G. (1997). The Mini International Neuropsychiatric Interview (MINI). A short diagnostic structured interview: Reliability and validity according to the CIDI. European Psychiatry, 12(5), 224-231. https://doi.org/10.1016/S0924-9338(97)83296-8

Luyten, P., Blatt, S. J., \& Fonagy, P. (2013). Impairments in self-structures in depression and suicide in psychodynamic and cognitive behavioral approaches: Implications for clinical practice and research. International Journal of Cognitive Therapy, 6(3), 265-279. https://doi.org/10.1521/ijct 2013.6.3.265

Luyten, P., Fonagy, P., Lemma, A., \& Target, M. (2019). Depression. In: A. W. Bateman \& P. Fonagy (Eds.), Handbook of mentalizing in mental health practice (pp. 387-402) American Psychiatric Accociation.

Luyten, P., Malcorps, S., Fonagy, P., \& Ensink, K. (2019). Assessment of mentalizing. In: A. W. Bateman \& P. Fonagy (Eds.), Handbook of mentalizing in mental health practice (pp. 37- 62). American Psychiatric Accociation.

Mund, M., \& Nestler, S. (2019). Beyond the Cross-Lagged Panel Model: Next-generation statistical tools for analyzing interdependencies across the life course. Advances in Life Course Research, 41, 100249. https:// doi.org/10.1016/j.alcr.2018.10.002

Muthén, B. O., \& Curran, P. J. (1997). General longitudinal modeling of individual differences in experimental designs: A latent variable framework for analysis and power estimation. Psychological Methods, 2(4), 371-402. https://doi.org/10.1037/1082-989X.2.4.371

Muthén, L. K., \& Muthén, B. O. (2018). Mplus 8.2. Muthén \& Muthén.
Owen, J., Adelson, J., Budge, S., Wampold, B., Kopta, M., Minami, T., \& Miller, S. (2015). Trajectories of change in psychotherapy. Journal of Clinical Psychology, 71, 817-827. https://doi.org/10.1002/jclp.22191

Rizzuto, A. M., McDargh, J., Aletti, M., Austad, A., Engedal, L. G., Stern, A., \& Stålsett, G. (2017). Ana-María Rizzuto and the psychoanalysis of religion: The road to the living God. Lexington Books.

Rost, F., Luyten, P., Fearon, P., \& Fonagy, P. (2019). Personality and outcome in individuals with treatment-resistant depression-exploring differential treatment effects in the Tavistock Adult Depression Study (TADS). Journal of Consulting and Clinical Psychology, 87(5), 433-445. https://doi.org/10.1037/ccp0000391

Saunders, J. B., Aasland, O. G., Babor, T. F., De la Fuente, J. R., \& Grant, M. (1993). Development of the alcohol use disorders identification test (AUDIT): WHO collaborative project on early detection of persons with harmful alcohol consumption-II. Addiction, 88(6), 791-804. https://doi.org/10.1111/j.1360-0443.1993.tb02093.x

Segal, Z. V., \& Teasdale, J. (2018). Mindfulness-based cognitive therapy for depression. Guilford Press.

Siqveland, J., \& Kornør, H. (2011). Måleegenskaper ved den norske versjonen av Beck Depression Inventory II (BDI II).

Slade, A., Aber, J. L., Bresgi, I., Berger, B., \& Kaplan, M. (2004). The parent development interview-revised [Unpublished protocol]. The City University of New York.

Sprinkle, S. D., Lurie, D., Insko, S. L., Atkinson, G., Jones, G. L., Logan, A. R., \& Bissada, N. N. (2002). Criterion validity, severity cut scores, and test-retest reliability of the Beck Depression Inventory-II in a university counseling center sample. Journal of Counseling Psychology, 49(3), 381-385. https://doi.org/10.1037/0022-0167.49.3.381

Stålsett, G. (2012). Existential and religious issues in psychotherapy: Development and evaluation of a new integrative treatment model (VITA) for comorbid depressive disorders [ $\mathrm{PhD}$ dissertation]. University of Oslo.

Taubner, S., Kessler, H., Buchheim, A., Kächele, H., \& Staun, L. (2011). The role of mentalization in the psychoanalytic treatment of chronic depression. Psychiatry, 74(1), 49-57. https://doi.org/10.1521/psyc.2011 .74 .1 .49

Wang, J., \& Wang, X. (2012). Structural equation modeling: Applications using Mplus. Wiley. https://doi.org/10.1002/9781118356258

Wang, L. P., \& Maxwell, S. E. (2015). On disaggregating between-person and within-person effects with longitudinal data using multilevel models. Psychological Methods, 20(1), 63-83. https://doi.org/10.1037/met0000030

Watkins, E., \& Teasdale, J. D. (2004). Adaptive and maladaptive self-focus in depression. Journal of Affective Disorders, 82(1), 1-8. https://doi.org/ 10.1016/j.jad.2003.10.006

Zilcha-Mano, S. (2017). Is the alliance really therapeutic? Revisiting this question in light of recent methodological advances. American Psychologist, 72(4), 311-325. https://doi.org/10.1037/a0040435

(Appendix follows) 
Appendix

\section{Mplus Analysis script for the Latent Growth Curve Model with Structured Residuals (LGCM-SR) for BDI-II and RFQ-C}

Mplus Syntax

Final Latent Growth Curve Model with Structured Residuals (LGCM-SR) for BDI-II and RFQ-C

Title: LGCM-SR model

.....

Variable:

Usevariables = BDI0 BDI1 BDI2 BDI3 BDI4 BDI5 BDI6 BDI7 BDI8 BDI9 BDI10 BDI11 BDI12

RFQc0 RFQc1 RFQc2 RFQc3 RFQc4 RFQc5 RFQc6 RFQc7 RFQc8

RFQc9 RFQc10 RFQc11 RFQc12

u0 u1 u2 u3 u4 u5 u6 u7 u8 u9 u10 u11 u12;

Tscores $=$ u0 u1 u2 u3 u4 u5 u6 u7 u8 u9 u10 u11 u12;

Analysis:

Estimator $=$ MLR;

Type = Random;

coverage $=.0001$;

!Processors $=4$;

model = nocovariances;

Model:

! BDI-

I S Q I BDI0-BDI12 AT u0-u12;

I with $S$ Q; $S$ with Q;

! Residuals as phantom variables (within level information)

e0 by BDI0@1; e1 by BDI1@1; e2 by BDI2@1; e3 by BDI3@1;

e4 by BDI4@1; e5 by BDI5@1; e6 by BDI6@1; e7 by BDI7@1;

e8 by BDI8@1; e9 by BDI9@1;

e10 by BDI10@1; e11 by BDI11@1; e12 by BDI12@1;

BDI0-BDI12@0;

[BDI0-BDI12@0];

[e0-e12@0];

e0-e12 (res1);

! RFQ - C

Ir Sr Qr I RFQc0-RFQc12 AT u0-u12;

Ir with Sr Qr; Sr with Qr;

! Residuals as phantom variables (within level information)

z0 by RFQc0@1; z1 by RFQc1@1; z2 by RFQc2@1; z3 by RFQc3@1;

z4 by RFQc4@1; z5 by RFQc5@1; z6 by RFQc6@1; z7 by RFQc7@1;

z8 by RFQc8@1; z9 by RFQc9@1;

z10 by RFQc10@1; z11 by RFQc11@1; z12 by RFQc12@1;

RFQc0-RFQc12@0;

[RFQc0-RFQc12@0];

[z0-z12@0];

z0-z12 (res2); 
! Relations (between effects)

I on Ir;

$\mathrm{S}$ on Ir Sr Qr;

$\mathrm{Q}$ on Ir Sr Qr;

! Residual relations (within effects)

e1-e12 pon e0-e11 (cross1); ! equal lag relations

z1-z12 pon z0-z11 (cross2); ! equal lag relations

$\mathrm{e} 0$ with $\mathrm{z} 0$;

e1-e12 pwith z1-z12 (eq1); ! equal residual covariance between e and z

e1-e12 pon z0-z11 (eq2); ! equal cross-lagged relations: e on $\mathrm{z}$

z1-z12 pon e0-e11 (eq3); ! equal cross-lagged relations: $z$ on e

Output:

Tech1;

Sampstat;

Standardized;

Received March 6, 2020

Revision received November 9, 2020

Accepted November 10, 2020 